\title{
Effect of Different Sources and Levels of K on Maize (Zea mays L.) Yield, Nutrient Content and Uptake by Maize Crop in Low K Soils of Eastern Dry Zone of Karnataka, India
}

\author{
Sidharam Patil ${ }^{*}$, P.K. Basavaraja ${ }^{1}$, V.R. Ramakrishna Parama ${ }^{1}$, \\ T. Chikkaramappa ${ }^{1}$ and T. Sheshadri ${ }^{2}$ \\ ${ }^{1}$ Department of Soil Science and Agricultural Chemistry, ${ }^{2}$ Department of Agronomy, University \\ of Agricultural Sciences, GKVK, Bangalore 560065, Karnataka, India \\ *Corresponding author
}

\section{A B S T R A C T}

Keywords

Maize, Potassium

Schoenite,

Quadratic

association.

Article Info

Accepted:

04 June 2017

Available Online:

10 August 2017
The present investigation was conducted to study the effect of different sources and levels of $\mathrm{K}$ on maize (Zea mays L.) yield, nutrient content and uptake by maize crop. Two field experiments were conducted in farmer's field at Sasalu and Sothenhalli villages of Dodaballapur taluk during kharif 2014 and 2015. Results indicated an increased grain and stover yield of maize with increase in levels of $\mathrm{K}_{2} \mathrm{O}$ application with significantly higher grain $\left(77.45 \mathrm{q} \mathrm{ha}^{-1}\right)$ and stover yield $\left(116.38 \mathrm{q} \mathrm{ha}^{-1}\right)$ recorded in $125 \%$ of $\mathrm{K}_{2} \mathrm{O}$ through potassium schoenite compared to Bio-K treatments. There was no significant difference in $\mathrm{N}, \mathrm{P}, \mathrm{K}$ content in grain and stover of maize crop due to different sources and levels of K. But, significantly higher total $\mathrm{N}$ uptake $\left(187.54 \mathrm{~kg} \mathrm{ha}^{-1}\right)$, total $\mathrm{P}$ uptake $\left(45.36 \mathrm{~kg} \mathrm{ha}^{-1}\right)$ and total $\mathrm{K}$ uptake $\left(148.94 \mathrm{~kg} \mathrm{ha}^{-1}\right)$ were recorded when $125 \%$ of $\mathrm{K}_{2} \mathrm{O}$ was applied through potassium schoenite and there was a positive quadratic association between levels of $\mathrm{K}_{2} \mathrm{O}$ application and NPK uptake by maize crop.

\section{Introduction}

Potassium is an essential nutrient element for all living organisms including plants and animals and its importance in Indian agriculture has increased with intensification of agriculture. It is a univalent cation found in largest concentration in the cell sap and hence it is called a "master cation". It plays vital roles in enzyme activation, water relations (osmotic regulations etc.), energy relations, translocation of assimilates, photosynthesis and, protein and starch synthesis (Mengel and Krikby, 1978).
Potassium is most pertinent nutrient that has the potential of significantly affecting agricultural production in the country. Historically low application rates of $\mathrm{K}$ in crops have led to over-depended on the native soil reserve of K (Sarkar et al., 2014). However, there is no reserve of K-bearing minerals in India for production of commercial K-fertilizers and the whole consumption of $\mathrm{K}$-fertilizers are imported in the form of muriate of potash $(\mathrm{KCl})$ and sulphate of potash $\left(\mathrm{K}_{2} \mathrm{SO}_{4}\right)$ which leads to a 
huge amount of foreign exchange. These necessitate finding an alternate indigenous source of $\mathrm{K}$ for plant needs and maintaining $\mathrm{K}$ status in soils for sustaining crop production.

Bio-K, a brown coloured powder is a value added product of distillery industry, where untreated spent wash is spray dried at high temperature. It retains most of the nutrients of spent wash and has very high potassium content. Similarly, potassium schoenite, a double sulfate of potassium and magnesium is made by physical extraction method or by direct removing impurities either from salt lake bittern or solid potassium - magnesium salt mine. These two can be used as a source of $\mathrm{K}$.

Maize has a high production potential as an exhaustive crop for potassium fertilizer when compared to any other cereal crop. Moreover, productivity of maize largely depends on its nutrient requirement. Large quantity of potassium will be taken up by maize crop, which accounts to more than $400 \mathrm{~kg} \mathrm{~K}_{2} \mathrm{O} \mathrm{ha}^{-1}$ under intensive cropping system (Kusro et al., 2014). So, with this background present study to know the effect of different sources and levels of $\mathrm{K}$ on yield, content and uptake of major nutrients by maize crop in Eastern dry zone of Karnataka was carried out.

\section{Materials and Methods}

Two field experiments were conducted in low $\mathrm{K}$ soils of Dodaballapur taluk in farmer's field at Saslu (Site-1) and Sothenhalli (Site-2) villages of Bangalore rural district during 2014-15 and 2015-16. Fields were located at $13^{\circ} 24^{\prime} 27.5^{\prime}$ ' $\mathrm{N}$ latitude, $77^{\circ} 23^{\prime} 11.3^{\prime}$ ' E longitude and $13^{\circ} 23^{\prime} 16.7^{\prime}, \mathrm{N}$ latitude, $77^{\circ} 34^{\prime} 45.5^{\prime}$ ' E longitude. The experiments were laid out in a factorial randomized complete block design with one control i.e., 3 sources and 4 levels of $\mathrm{K}_{2} \mathrm{O}$ with one control which were replicated thrice. The soils of the experimental fields' were acidic in $\mathrm{pH}$ (5.26 and 5.04) and initial physico-chemical properties of soils are presented in table 1 . Farm yard manure $\left(10 \mathrm{t} \mathrm{ha}^{-1}\right)$ was incorporated 15 days prior to sowing, maize seeds were dibbled at a spacing of $60 \mathrm{~cm} \times 30$ $\mathrm{cm}$ and recommended dose of $\mathrm{N}, \mathrm{P}$ and $\mathrm{ZnSO}_{4}$ (150, 75 and $10 \mathrm{~kg} \mathrm{ha}^{-1}$, respectively.) with varied levels of $\mathrm{K}_{2} \mathrm{O}$ i.e., 50, 75, 100 and 125 per cent of recommended dose of $\mathrm{K}_{2} \mathrm{O}$ were applied as per the package of practice of UAS, Bangalore and as per the treatments at the time of sowing. All other recommended agronomic practices were carried out to all treatments uniformly during the course of the study.

At harvest five plant samples including cobs were randomly collected from each treatment and washed with tap water and finally with distilled water to remove the adhering soil and dusts. Then they were air dried and later kept in the hot air oven at $60-70^{\circ} \mathrm{C}$ for eight hours. The oven dried samples were powdered separately for grain and stover with willey milling machine and stored in polythene bags. These samples were analyzed for nitrogen, phosphorus and potassium content following standard procedures i.e., nitrogen concentration was determined by Kjeldahl's digestion-distillation method as described by Piper (1966), phosphorus was determination by vanadomolybdophosphoric yellow colour method as described by Jackson (1973), potassium was determined using flame photometer as described by Piper (1966) and uptake of respective nutrients was calculated by taking into consideration of biological yield. The data recorded on various parameters was statistically analyzed as described by Gomez and Gomez (1984). The trend in yield, nutrient concentration and uptake of nutrients remained same during both the years; as such pooled mean over the two years of experimental data has been presented and discussed. 


\section{Results and Discussion}

\section{Grain and stover yield}

Irrespective of $\mathrm{K}$ levels, significant difference in grain and stover yield of maize was recorded due to different sources of $\mathrm{K}$ application (Table 2). Significantly higher grain and stover yield of $63.29 \mathrm{q} \mathrm{ha}^{-1}$ and $95.01 \mathrm{q} \mathrm{ha}^{-1}$, respectively were recorded in potassium schoenite $\left(\mathrm{S}_{2}\right)$ applied treatment compared to MOP $\left(\mathrm{S}_{1}\right)$ and Bio-K $\left(\mathrm{S}_{3}\right)$ applied treatment. On the other hand, among the different levels of $\mathrm{K}_{2} \mathrm{O}$ applied, irrespective of $\mathrm{K}$ sources, there was an increase in grain and stover yield parallel to increase in levels of $\mathrm{K}_{2} \mathrm{O}$ applied with significantly higher grain $\left(70.99 \mathrm{q} \mathrm{ha}^{-1}\right)$ and stover yield $\left(105.58 \mathrm{q} \mathrm{ha}^{-1}\right)$ in $125 \%$ of recommended $\mathrm{K} \quad\left(\mathrm{L}_{4}\right)$ applied treatment compared to all other levels of $\mathrm{K}$ applied.

Among the interaction between different sources and levels of $\mathrm{K}$, significantly higher grain and stover yield of $77.45 \mathrm{q} \mathrm{ha}^{-1}$ and $116.38 \mathrm{q} \mathrm{ha}^{-1}$, respectively was recorded in $125 \%$ of recommended $\mathrm{K}_{2} \mathrm{O}$ applied through potassium schoenite $\left(\mathrm{S}_{2} \mathrm{~L}_{4}\right)$ compared to control and all other treatments except $125 \%$ of recommended $\mathrm{K}_{2} \mathrm{O}$ through Bio- $\mathrm{K}$ with $71.98 \mathrm{q} \mathrm{ha}^{-1}$ of grain yield and $100 \% \mathrm{~K}_{2} \mathrm{O}$ through potassium schoenite in case of stover yield $\left(107.00 \mathrm{q} \mathrm{ha}^{-1}\right)$ which were statistically on par.

Increase in growth and yield parameters of maize crop with increased levels of $\mathrm{K}_{2} \mathrm{O}$ application might be due to increased physiological processes by better utilization of applied NPK fertilizers by maize crop leading to higher plant growth and increased photosynthates to silk as the translocation and accumulation of photosynthates depends upon the efficient photosynthetic structure as well as extent of trans locating it into sink (grain) and also on plant growth and development during early stage of crop growth (Arunkumar et al., 2007).

Increased grain and stover yield in $125 \%$ of recommended $\mathrm{K}_{2} \mathrm{O}$ through potassium schoenite compared to that of MOP and Bio$\mathrm{K}$ as source of $\mathrm{K}$, might be due to $\mathrm{S}$ content in the potassium schoenite which might have increased nitrogen assimilation thereby increasing grain and stover yield. The results are in conformity with those of Manjunath (2011), who found increase in growth and yield parameters of rice crop in patent kali $\left(\mathrm{K}_{2} \mathrm{SO}_{4} . \mathrm{MgSO}_{4}\right)$ applied plot which resulted in higher grain and straw yield attributed it to increased rate of photosynthesis and trans locating it to sink (grain). Similarly, increase in grain and stover yield of maize crop with higher levels of sulphur application was reported by Channabasamma et al., (2013) and it was attributed to greater rate of cell division, nitrogen assimilation and chlorophyll formation.

\section{Nutrient concentration}

There was no significant difference in primary nutrients content in grain and stover of maize crop mainly because of the dilution effect of nutrient concentration due to higher biomass yield from the applied nutrients (Table 3).

\section{Nutrient uptake}

Trend in uptake of major nutrients by maize grain and stover remained same during both the years under different treatments. So, total uptake (grain + stover) is presented (Table 4 ) and discussed.

\section{Total uptake of nitrogen}

Total uptake of $\mathrm{N}$ by maize crop differed significantly with different sources of $\mathrm{K}$ applied, irrespective of levels of $\mathrm{K}$ applied. 
Significantly higher total N uptake $(149.65 \mathrm{~kg}$ $\mathrm{ha}^{-1}$ ) was recorded when potassium schoenite was applied $\left(\mathrm{S}_{2}\right)$ compared to that of MOP (129.25 kg ha ${ }^{-1}$ ) and Bio-K (141.26 kg ha-1) $\left(\mathrm{S}_{1}\right.$ and $\left.\mathrm{S}_{3}\right)$.

There was an increase in uptake of $\mathrm{N}$ with increase in levels of $\mathrm{K}$ applied, irrespective of sources of $\mathrm{K}$ applied. Significantly higher total $\mathrm{N}$ uptake $\left(170.69 \mathrm{~kg} \mathrm{ha}^{-1}\right)$ was recorded at $125 \%$ of recommended $\mathrm{K}_{2} \mathrm{O}$ application $\left(\mathrm{L}_{4}\right)$ compared to all other levels of $\mathrm{K}$ applied.

Interactions between different sources and levels of $\mathrm{K}$ application had a significant difference, where significantly higher $\mathrm{N}$ uptake $\left(187.54 \mathrm{~kg} \mathrm{ha}^{-1}\right)$ was recorded with $125 \%$ of recommended $\mathrm{K}_{2} \mathrm{O}$ through potassium schoenite $\left(\mathrm{S}_{2} \mathrm{~L}_{4}\right)$. However, there was no significant difference among the different sources at $50 \%$ and $75 \%$ of $\mathrm{K}_{2} \mathrm{O}$ application.

\section{Total uptake of $\mathbf{P}$}

Among the different sources of $\mathrm{K}$ irrespective of levels of $\mathrm{K}$ applied, significantly higher total $\mathrm{P}$ uptake by maize crop was recorded in potassium schoenite $\left(\mathrm{S}_{2}\right)$ applied plots $(36.17$ $\mathrm{kg} \mathrm{ha}^{-1}$ ) compared to that of MOP and Bio-K applied plots (31.73 and $34.17 \mathrm{~kg} \mathrm{ha}^{-1}$, respectively).

Similarly, significant difference in $\mathrm{P}$ uptake was recorded among the different levels of $\mathrm{K}$ applied irrespective of the $\mathrm{K}$ sources. Increase in $\mathrm{P}$ uptake was correspondingly to increase in levels of $\mathrm{K}$ applied. Significantly higher uptake of $\mathrm{P}$ (41.72 $\mathrm{kg} \mathrm{ha}^{-1}$ ) was recorded at $125 \%$ of recommended $\mathrm{K}_{2} \mathrm{O}\left(\mathrm{L}_{4}\right)$ compared to all other levels of $\mathrm{K}_{2} \mathrm{O}$ applied.

Perusal of $\mathrm{P}$ uptake data clearly indicated that there was a significant interaction between different sources and levels of $\mathrm{K}$ applied.

Table.1 Initial physico-chemical properties of soils in study area

\begin{tabular}{|c|c|c|}
\hline Parameters & Site-1 & Site-2 \\
\hline Location & Saslu & Sothenahalli \\
\hline \multicolumn{3}{|c|}{ Particle size distribution (\%) } \\
\hline Sand $(\%)$ & 74.4 & 68.8 \\
\hline Silt (\%) & 7.6 & 9.4 \\
\hline Clay (\%) & 18.0 & 21.8 \\
\hline Soil texture & Sandy loam & Sandy clay loam \\
\hline Bulk density $\left(\mathrm{g} \mathrm{cm}^{-3}\right)$ & 1.59 & 1.52 \\
\hline pH (1: 2.5) & 5.26 & 5.04 \\
\hline $\mathrm{EC}\left(\mathrm{dSm}^{-1}\right)$ & 0.161 & 0.054 \\
\hline $\mathrm{OC}(\%)$ & 0.46 & 0.38 \\
\hline $\operatorname{CEC}\left(\mathrm{cmol}\left(\mathrm{p}^{+}\right) \mathrm{kg}^{-1}\right)$ & 8.6 & 6.71 \\
\hline Available N $\left(\mathrm{kg} \mathrm{ha}^{-1}\right)$ & 298.54 & 322.38 \\
\hline Available $\mathrm{P}_{2} \mathrm{O}_{5}\left(\mathrm{~kg} \mathrm{ha}^{-1}\right)$ & 72.09 & 22.18 \\
\hline Available $\mathrm{K}_{2} \mathrm{O}\left(\mathrm{kg} \mathrm{ha}^{-1}\right)$ & 97.20 & 112.8 \\
\hline Available $S\left(\mathrm{mg} \mathrm{kg}^{-1}\right)$ & 11.02 & 8.24 \\
\hline $\mathrm{Ca}\left(\mathrm{c} \mathrm{mol}(\mathrm{p}+) \mathrm{kg}^{-1}\right)$ & 1.75 & 3.75 \\
\hline $\operatorname{Mg}\left(\mathrm{c} \mathrm{mol}(\mathrm{p}+) \mathrm{kg}^{-1}\right)$ & 0.75 & 1.50 \\
\hline $\mathrm{Zn}\left(\mathrm{mg} \mathrm{kg}^{-1}\right)$ & 1.38 & 1.27 \\
\hline $\operatorname{Mn}\left(\mathrm{mg} \mathrm{kg}^{-1}\right)$ & 8.74 & 7.68 \\
\hline $\mathrm{Fe}\left(\mathrm{mg} \mathrm{kg}^{-1}\right)$ & 14.71 & 16.37 \\
\hline $\mathrm{Cu}\left(\mathrm{mg} \mathrm{kg}^{-1}\right)$ & 0.79 & 0.83 \\
\hline$B\left(\mathrm{mg} \mathrm{kg}^{-1}\right)$ & 0.57 & 0.28 \\
\hline
\end{tabular}


Table.2 Effect of different sources and levels of potassium on grain and Stover yield ( $\mathrm{q} \mathrm{ha}^{-1}$ ) of maize crop (pooled over 2 years)

\begin{tabular}{|c|c|c|c|c|c|c|}
\hline \multirow[b]{2}{*}{ Treatments } & \multicolumn{3}{|c|}{ Grain yield $\left(q\right.$ ha $\left.^{-1}\right)$} & \multicolumn{3}{|c|}{ Stover yield $\left(q\right.$ ha $\left.^{-1}\right)$} \\
\hline & $\begin{array}{c}\text { Kharif } \\
2014\end{array}$ & $\begin{array}{c}\text { Kharif } \\
2015\end{array}$ & $\begin{array}{c}\text { Pooled } \\
\text { mean }\end{array}$ & $\begin{array}{c}\text { Kharif } \\
2014\end{array}$ & $\begin{array}{c}\text { Kharif } \\
2015\end{array}$ & $\begin{array}{c}\text { Pooled } \\
\text { mean }\end{array}$ \\
\hline $\mathrm{S}_{1}: \mathrm{MOP}$ & 53.73 & 55.87 & 54.80 & 83.23 & 87.47 & 85.35 \\
\hline$S_{2}$ : Pot. Schoenite & 62.12 & 64.45 & 63.29 & 92.88 & 97.13 & 95.01 \\
\hline $\mathbf{S}_{3}$ : Bio-K & 58.30 & 61.70 & 60.00 & 84.68 & 91.86 & 88.27 \\
\hline SEm \pm & 1.75 & 1.19 & 1.05 & 3.05 & 2.18 & 2.17 \\
\hline CD@5\% & 5.10 & 3.47 & 2.96 & 8.91 & 6.35 & 6.12 \\
\hline $\mathrm{L}_{1}: 50 \% \mathrm{~K}$ & 45.25 & 50.77 & 48.01 & 65.22 & 80.11 & 72.66 \\
\hline $\mathrm{L}_{2}: 75 \% \mathrm{~K}$ & 53.24 & 54.50 & $\mathbf{5 3 . 8 7}$ & 73.18 & 87.87 & 80.53 \\
\hline $\mathrm{L}_{3}: 100 \% \mathrm{~K}$ & 64.19 & 64.96 & 64.58 & 100.98 & 97.80 & 99.39 \\
\hline $\mathrm{L}_{4}: 125 \% \mathrm{~K}$ & 69.52 & 72.46 & 70.99 & 108.33 & 102.84 & 105.58 \\
\hline SEm \pm & 2.02 & 1.37 & 1.21 & 3.53 & 2.51 & 2.50 \\
\hline CD@5\% & 5.88 & 4.00 & 3.42 & 10.29 & 7.34 & 7.07 \\
\hline $\mathrm{S}_{1} \mathrm{~L}_{1}: 50 \% \mathrm{~K}(\mathrm{MOP})$ & 42.60 & 48.19 & 45.40 & 63.30 & 75.72 & 69.51 \\
\hline$S_{1} L_{2}: 75 \%$ K (MOP) & 52.43 & 51.11 & 51.77 & 72.41 & 86.74 & 79.58 \\
\hline$S_{1} L_{3}: 100 \%$ K (MOP) & 58.18 & 58.77 & 58.48 & 93.54 & 93.44 & 93.49 \\
\hline$S_{1} L_{4}: 125 \%$ K (MOP) & 61.70 & 65.41 & 63.56 & 103.66 & 93.99 & 98.82 \\
\hline $\mathrm{S}_{2} \mathrm{~L}_{1}: 50 \% \mathrm{~K}$ (Pot. schoenite) & 47.20 & 52.95 & 50.08 & 67.37 & 82.63 & 75.00 \\
\hline $\mathrm{S}_{2} \mathrm{~L}_{2}: 75 \% \mathrm{~K}$ (Pot. schoenite) & 55.63 & 58.25 & 56.94 & 73.64 & 89.66 & 81.65 \\
\hline$S_{2} L_{3}: 100 \%$ K (Pot. schoenite) & 68.48 & 68.88 & 68.68 & 112.85 & 101.14 & 107.00 \\
\hline $\mathrm{S}_{2} \mathrm{~L}_{4}: 125 \% \mathrm{~K}$ (Pot. schoenite) & 77.18 & 77.72 & 77.45 & 117.66 & 115.11 & 116.38 \\
\hline$S_{3} L_{1}: 50 \%$ K (Bio-K) & 45.95 & 51.15 & 48.55 & 64.98 & 81.96 & 73.47 \\
\hline $\mathrm{S}_{3} \mathrm{~L}_{2}: 75 \% \mathrm{~K}($ Bio-K) & 51.65 & 54.15 & 52.90 & 73.50 & 87.23 & 80.36 \\
\hline$S_{3} L_{3}: 100 \%$ K (Bio-K) & 65.91 & 67.24 & 66.57 & 96.55 & 98.84 & 97.69 \\
\hline$S_{3} L_{4}: 125 \%$ K (Bio-K) & 69.70 & 74.26 & 71.98 & 103.67 & 99.42 & 101.54 \\
\hline $\mathrm{S}_{0} \mathrm{~L}_{0}:$ Control (No-K) & 36.68 & 38.96 & 37.82 & 41.82 & 53.92 & 47.87 \\
\hline SEm \pm & 3.49 & 2.38 & 2.10 & 6.11 & 4.35 & 4.33 \\
\hline CD@5\% & 10.19 & 6.94 & 5.92 & 17.82 & 12.71 & 12.25 \\
\hline
\end{tabular}

*S- Source, L- Levels of $\mathrm{K},\left(\mathrm{N}, \mathrm{P}, \mathrm{ZnSO}_{4}\right.$ and $\mathrm{FYM}$ were applied as per package of practice to all the treatments) 
Table.3 Effect of different sources and levels of $\mathrm{K}$ on $\mathrm{N}, \mathrm{P}$ and $\mathrm{K}$ content in maize Stover and grain (pooled over 2 years)

\begin{tabular}{|c|c|c|c|c|c|c|}
\hline \multirow[b]{2}{*}{ Treatments } & \multicolumn{2}{|c|}{$\% \mathrm{~N}$} & \multicolumn{2}{|c|}{$\% \mathbf{P}$} & \multicolumn{2}{|c|}{$\% \mathrm{~K}$} \\
\hline & Stover & Grain & Stover & Grain & Stover & Grain \\
\hline $\mathrm{S}_{1}: \mathrm{MOP}$ & 0.74 & 1.57 & 0.20 & 0.37 & 1.30 & 0.53 \\
\hline$S_{2}$ : Pot. Schoenite & 0.76 & 1.59 & 0.20 & 0.37 & 1.32 & 0.53 \\
\hline $\mathrm{S}_{3}$ : Bio-K & 0.76 & 1.59 & 0.20 & 0.37 & 1.33 & 0.53 \\
\hline SEm \pm & 0.01 & 0.02 & 0.00 & 0.00 & NS & 0.01 \\
\hline CD@5\% & NS & NS & NS & NS & 0.08 & NS \\
\hline $\mathrm{L}_{1}: 50 \% \mathrm{~K}$ & 0.74 & 1.57 & 0.19 & 0.37 & 1.29 & 0.52 \\
\hline $\mathrm{L}_{2}: 75 \% \mathrm{~K}$ & 0.76 & 1.58 & 0.19 & 0.37 & 1.34 & 0.53 \\
\hline $\mathrm{L}_{3}: 100 \% \mathrm{~K}$ & 0.74 & 1.57 & 0.20 & 0.37 & 1.30 & 0.53 \\
\hline $\mathrm{L}_{4}: 125 \% \mathrm{~K}$ & 0.77 & 1.63 & 0.20 & 0.38 & 1.34 & 0.54 \\
\hline SEm \pm & 0.02 & 0.02 & 0.01 & 0.01 & 0.03 & 0.01 \\
\hline CD@5\% & NS & $\mathbf{N S}$ & NS & 0.01 & NS & NS \\
\hline$S_{1} L_{1}: 50 \%$ K (MOP) & 0.73 & 1.56 & 0.19 & 0.38 & 1.27 & 0.52 \\
\hline$S_{1} L_{2}: 75 \%$ K (MOP) & 0.75 & 1.56 & 0.19 & 0.37 & 1.32 & 0.52 \\
\hline$S_{1} L_{3}: 100 \%$ K (MOP) & 0.74 & 1.56 & 0.20 & 0.37 & 1.30 & 0.54 \\
\hline$S_{1} L_{4}: 125 \%$ K (MOP) & 0.76 & 1.62 & 0.20 & 0.38 & 1.33 & 0.55 \\
\hline$S_{2} L_{1}: 50 \%$ K (Pot. Schoenite) & 0.75 & 1.56 & 0.19 & 0.38 & 1.28 & 0.52 \\
\hline$S_{2} L_{2}: 75 \%$ K (Pot. Schoenite) & 0.76 & 1.60 & 0.20 & 0.37 & 1.35 & 0.52 \\
\hline $\mathbf{S}_{2} \mathbf{L}_{3}: 100 \%$ K (Pot. Schoenite) & 0.75 & 1.57 & 0.19 & 0.37 & 1.29 & 0.53 \\
\hline$S_{2} L_{4}: 125 \%$ K (Pot. Schoenite) & 0.78 & 1.63 & 0.20 & 0.38 & 1.36 & 0.54 \\
\hline$S_{3} L_{1}: 50 \%$ K (Bio- K) & 0.75 & 1.58 & 0.20 & 0.37 & 1.32 & 0.52 \\
\hline$S_{3} L_{2}: 75 \%$ K (Bio- K) & 0.77 & 1.61 & 0.19 & 0.37 & 1.35 & 0.54 \\
\hline $\mathrm{S}_{3} \mathrm{~L}_{3}: 100 \% \mathrm{~K}(\mathrm{Bio}-\mathrm{K})$ & 0.75 & 1.57 & 0.20 & 0.36 & 1.31 & 0.52 \\
\hline $\mathrm{S}_{3} \mathrm{~L}_{4}: \mathbf{1 2 5 \%} \mathrm{K}(\mathrm{Bio}-\mathrm{K})$ & 0.78 & 1.63 & 0.21 & 0.38 & 1.34 & 0.54 \\
\hline $\mathbf{S}_{0} \mathbf{L}_{0}:$ Control K (No-K) & 0.72 & 1.56 & 0.19 & 0.37 & 1.33 & 0.51 \\
\hline SEm \pm & $\mathbf{0 . 0 3}$ & 0.04 & 0.01 & 0.01 & 0.06 & 0.02 \\
\hline CD@5\% & NS & NS & NS & NS & NS & $\mathbf{N S}$ \\
\hline
\end{tabular}

*S- Source, $\mathrm{L}$ - Levels of $\mathrm{K},\left(\mathrm{N}, \mathrm{P}, \mathrm{ZnSO}_{4}\right.$ and $\mathrm{FYM}$ were applied as per package of practice to all the treatments) 
Table.4 Effect of different sources and levels of K on total N, P and K uptake by maize (Pooled over 2 years)

\begin{tabular}{|c|c|c|c|c|c|c|c|c|c|}
\hline \multirow[b]{2}{*}{ Treatments } & \multicolumn{3}{|c|}{ N uptake $\left(\mathrm{kg} \mathrm{ha}^{-1}\right)$} & \multicolumn{3}{|c|}{ P uptake $\left(\mathrm{kg} \mathrm{ha}^{-1}\right)$} & \multicolumn{3}{|c|}{ K uptake $\left(\mathrm{kg} \mathrm{ha}^{-1}\right)$} \\
\hline & Stover & Grain & Total & Stover & Grain & Total & Stover & Grain & Total \\
\hline $\mathrm{S}_{1}:$ MOP & 42.82 & 86.96 & 129.25 & 11.30 & 20.43 & 31.73 & 75.13 & 29.26 & 104.39 \\
\hline$S_{2}:$ Pot. Schoenite & 48.65 & 101.00 & 149.65 & 12.59 & 23.58 & 36.17 & 84.98 & 33.60 & 118.59 \\
\hline$S_{3}:$ Bio-K & 45.23 & 96.03 & 141.26 & 11.73 & 22.44 & 34.17 & 78.94 & 31.75 & 110.69 \\
\hline SEm \pm & 1.16 & 2.07 & 2.74 & 0.32 & 0.46 & 0.59 & 2.53 & $\mathbf{0 . 8 3}$ & $\mathbf{3 . 0 3}$ \\
\hline CD@5\% & 3.27 & 5.84 & 7.75 & 0.92 & 1.31 & 1.68 & 7.16 & 2.35 & 8.55 \\
\hline $\mathrm{L}_{1}: \mathbf{5 0} \% \mathrm{~K}$ & 36.20 & 75.25 & 111.45 & 9.31 & 17.88 & 27.19 & 62.92 & 25.01 & $\mathbf{8 7 . 9 3}$ \\
\hline $\mathrm{L}_{2}: 75 \% \mathrm{~K}$ & 41.08 & 85.85 & 126.90 & 10.52 & 19.93 & 30.45 & 72.72 & 28.39 & 101.11 \\
\hline $\mathrm{L}_{3}: 100 \% \mathrm{~K}$ & 49.86 & 101.30 & 151.16 & 13.09 & 23.64 & 36.73 & 86.99 & 34.15 & 121.14 \\
\hline $\mathrm{L}_{4}: 125 \% \mathrm{~K}$ & 55.12 & 115.57 & 170.69 & 14.57 & 27.15 & 41.72 & 96.11 & 38.59 & 134.70 \\
\hline SEm \pm & 1.34 & 2.39 & 3.17 & $\mathbf{0 . 3 7}$ & 0.54 & 0.69 & 2.93 & 0.96 & 3.49 \\
\hline CD @5\% & 3.77 & 6.74 & 8.95 & 1.06 & 1.52 & 1.94 & 8.27 & 2.71 & 9.87 \\
\hline$S_{1} L_{1}: 50 \%$ K (MOP) & 34.16 & 70.76 & 104.92 & 8.84 & 17.04 & 25.88 & 59.39 & 23.66 & 83.05 \\
\hline$S_{1} L_{2}: 75 \%$ K (MOP) & 39.90 & 80.93 & 120.82 & 10.28 & 18.95 & 29.23 & 70.47 & 26.78 & 97.25 \\
\hline$S_{1} L_{3}: 100 \%$ K (MOP) & 46.44 & 90.93 & 137.37 & 12.43 & 21.58 & 34.01 & 81.39 & 31.60 & 112.99 \\
\hline$S_{1} L_{4}: 125 \%$ K (MOP) & 50.77 & 103.12 & 153.89 & 13.66 & 24.15 & 37.81 & 89.28 & 34.97 & 124.24 \\
\hline $\begin{array}{l}\mathrm{S}_{2} \mathbf{L}_{1}: 50 \% \text { K (Pot. } \\
\text { Schoenite) }\end{array}$ & 37.46 & 78.27 & 115.73 & 9.62 & 18.75 & 28.37 & 65.24 & 26.17 & 91.41 \\
\hline $\begin{array}{l}S_{2} \mathbf{L}_{2}: 75 \% \text { K (Pot. } \\
\text { Schoenite) }\end{array}$ & 42.05 & 91.14 & 133.19 & 10.84 & 20.96 & 31.81 & 74.34 & 29.76 & 104.10 \\
\hline $\begin{array}{l}S_{2} \mathbf{L}_{3}: 100 \% \text { K (Pot. } \\
\text { Schoenite) }\end{array}$ & 53.96 & 108.16 & 162.13 & 14.01 & 25.15 & 39.15 & 93.29 & 36.60 & 129.89 \\
\hline $\begin{array}{l}S_{2} \mathbf{L}_{4}: 125 \% \mathrm{~K} \text { (Pot. } \\
\text { Schoenite) }\end{array}$ & 61.11 & 126.43 & 187.54 & 15.89 & 29.47 & 45.36 & 107.06 & 41.88 & 148.94 \\
\hline $\mathrm{S}_{3} \mathbf{L}_{1}: 50 \% \mathrm{~K}($ Bio- K $)$ & 36.98 & 76.71 & 113.69 & 9.47 & 17.85 & 27.32 & 64.14 & 25.20 & 89.34 \\
\hline$S_{3} L_{2}: 75 \% \mathrm{~K}($ Bio- $K)$ & 41.29 & 85.48 & 126.77 & 10.45 & 19.89 & 30.33 & 73.33 & 28.63 & 101.96 \\
\hline$S_{3} L_{3}: 100 \%$ K (Bio- K) & 49.18 & 104.81 & 153.98 & 12.84 & 24.19 & 37.03 & 86.28 & 34.26 & 120.54 \\
\hline$S_{3} L_{4}: 125 \%$ K (Bio- K) & 53.48 & 117.15 & 170.63 & 14.17 & 27.83 & 42.00 & 92.00 & 38.92 & 130.92 \\
\hline $\mathrm{S}_{0} \mathrm{~L}_{0}:$ Control K (No-K) & 23.33 & 58.81 & 82.14 & 6.10 & 13.75 & 19.85 & 43.06 & 19.41 & 62.47 \\
\hline $\mathrm{SEm} \pm$ & 2.31 & 4.13 & 5.48 & 0.65 & 0.93 & 1.19 & 5.07 & 1.66 & 6.05 \\
\hline CD@5\% & 6.54 & 11.68 & 15.40 & 1.83 & 2.62 & 3.36 & 14.32 & 4.69 & $\mathbf{1 7 . 1 0}$ \\
\hline
\end{tabular}

${ }^{*} \mathrm{~S}$ - Source, L- Levels of $\mathrm{K},\left(\mathrm{N}, \mathrm{P}, \mathrm{ZnSO}_{4}\right.$ and FYM were applied as per package of practice to all the treatments) 
Fig.1 Relationship between total $\mathrm{N}$ uptake and levels of $\mathrm{K}$

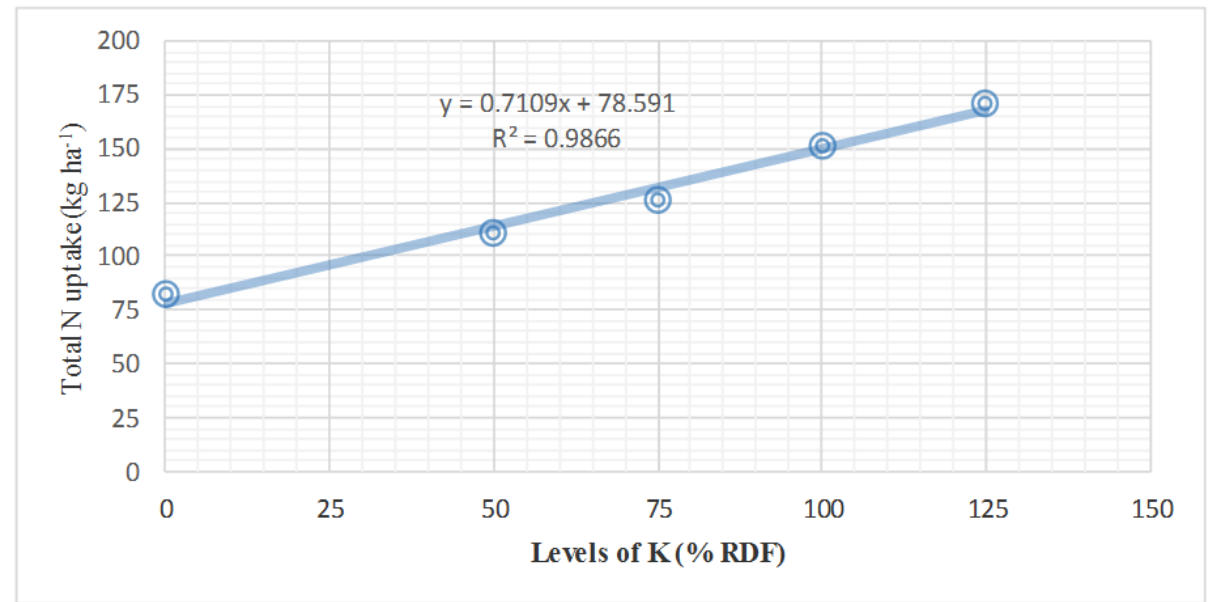

Fig.2 Relationship between total P uptake and levels of K

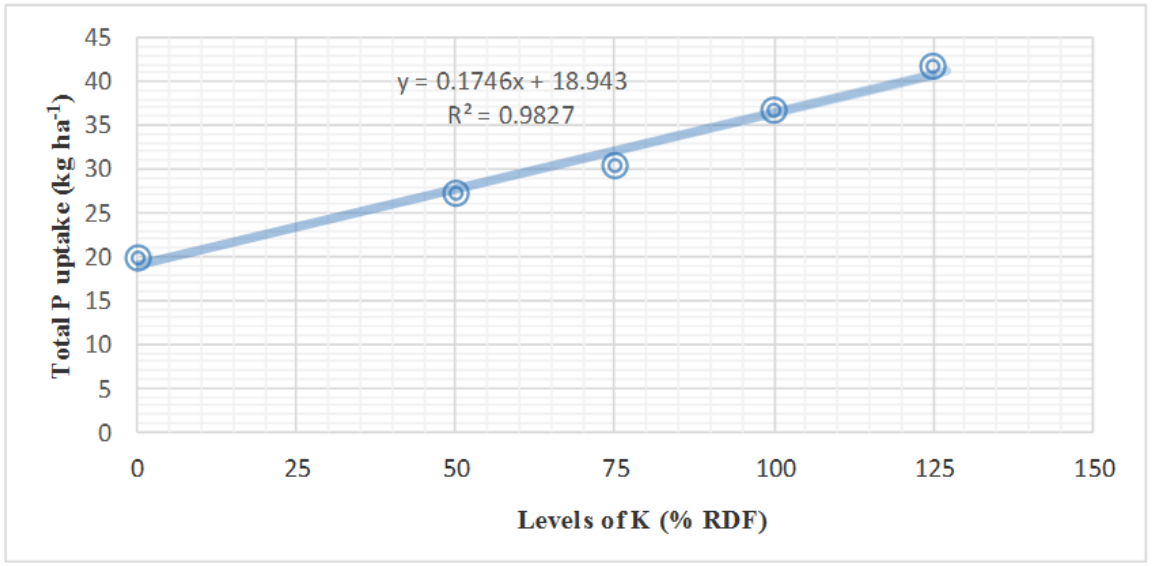

Fig.3 Relationship between total K uptake and levels of K

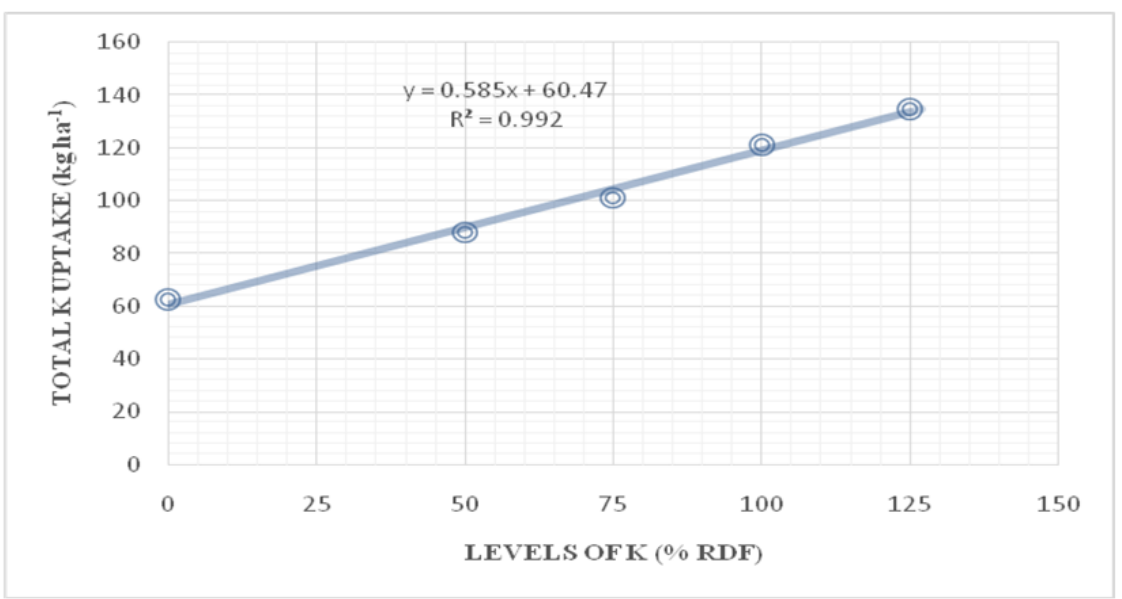


Significantly higher $45.36 \mathrm{~kg} \mathrm{ha}^{-1} \mathrm{P}$ uptake was recorded at $125 \%$ of recommended $\mathrm{K}_{2} \mathrm{O}$ application as potassium schoenite $\left(\mathrm{S}_{2} \mathrm{~L}_{4}\right)$ compared to all other treatments, except when $125 \%$ of recommended $\mathrm{K}_{2} \mathrm{O}$ was applied as Bio-K $\left(\mathrm{S}_{3} \mathrm{~L}_{4}\right)$, which was statistically on par. However, there was no significant difference at $50 \%$ and $75 \%$ of $\mathrm{K}_{2} \mathrm{O}$ applied irrespective of $\mathrm{K}$ sources.

\section{Total uptake of $\mathrm{K}$}

Irrespective of $\mathrm{K}$ levels, there was a significant difference in $\mathrm{K}$ uptake by maize crop among the different sources of $\mathrm{K}$ applied, where significantly higher K uptake (118.59 $\mathrm{kg} \mathrm{ha}^{-1}$ ) was recorded with potassium schoenite $\left(\mathrm{S}_{2}\right)$ application compared to MOP $\left(\mathrm{S}_{1}\right)$. But, it was statistically on par with treatment receiving Bio-K (110.69 $\left.\mathrm{kg} \mathrm{ha}^{-1}\right)$ as source of K.

Similarly, significant difference in $\mathrm{K}$ uptake was recorded among the different levels of $\mathrm{K}$ applied irrespective of the $\mathrm{K}$ sources. There was an increasing trend in $\mathrm{K}$ uptake with concomitant increase in levels of $\mathrm{K}$ application, where significantly higher total uptake of $\mathrm{K}\left(134.70 \mathrm{~kg} \mathrm{ha}^{-1}\right)$ was recorded at $125 \%$ recommended $\mathrm{K}_{2} \mathrm{O}\left(\mathrm{L}_{4}\right)$ application compared to all other levels of $\mathrm{K}$ applied and lowest $\mathrm{K}$ uptake was recorded with $50 \%$ of $\mathrm{K}$ $\left(\mathrm{L}_{1}\right)$.

Among the interactions of sources and levels of $\mathrm{K}$ application, significant difference in total uptake of $\mathrm{K}$ was recorded with highest uptake $\left(148.94 \mathrm{~kg} \mathrm{ha}^{-1}\right)$ in $125 \%$ of recommended $\mathrm{K}_{2} \mathrm{O}$ application through potassium schoenite $\left(\mathrm{S}_{2} \mathrm{~L}_{4}\right)$ compared to other treatments. Significantly lower K uptake $\left(62.47 \mathrm{~kg} \mathrm{ha}^{-1}\right)$ was recorded in control plot where no $\mathrm{K}$ was applied.

Increased uptake of $\mathrm{N}, \mathrm{P}$ and $\mathrm{K}$ by maize crop with increased levels of $\mathrm{K}$ application might be due to synergetic interaction between $\mathrm{N}$ and $\mathrm{K}$, where nitrogen is a fundamental nutrient in production of proteins. Plants deficient in potassium will not produce proteins despite an abundance of available nitrogen. Instead, incomplete proteins such as amino acids, amides and nitrates will accumulate in the cell. This is because of the enzyme nitrate reductase, which catalyzes the formation of proteins, is activated by potassium (Ujwala Ranade, 2011).

Similar to the present study on maize crop, increase in uptake of different nutrients by wheat crop due to increased levels of potassium was noticed by Sanjeev Kumar et al., (2015), which was attributed to translocation of nutrients by applied $\mathrm{K}$ which plays a role in increasing nutrients uptake by plants. Potassium has a synergetic effect on uptake of nitrogen and other nutrients due to which all the treatments applied with potassium was superior to control. Similarly, Bagavatiammal and Muthiah, (1995) and Sudhir et al., (1998) reported that application of FYM along with recommended dose of fertilizer and with increased $\mathrm{K}$ rates recorded higher nutrient uptake by the crops. Similarly, application of fertilizer $\mathrm{K}$ in combination with $\mathrm{N}$ showed a synergetic influence on uptake, translocation and utilization of nutrients for growth and development, and increased grain yield in maize (Ahmad Alias Haji et al., 2012).

In the present study higher uptake of $\mathrm{N}, \mathrm{P}$ and $\mathrm{K}$ was recorded at $125 \%$ of recommended $\mathrm{K}_{2} \mathrm{O}$ applied through potassium schoenite. This was due to presence of sulphur in potassium schoenite, which might have influenced the $\mathrm{N}$ assimilation, thereby increasing the dry matter production. Further, sulphur contributed by potassium schoenite and single super phosphate might have influenced higher $\mathrm{P}$ uptake. However, the main reason may be due to the synergetic 
effect of P and S. Similar results were recorded by Shubhangi et al., (2014) who found that uptake of phosphorus and sulphur increased with increase in the rate of application of $\mathrm{P}$ and $S$ individually as well as in various combinations and attributed it to synergetic effect between $\mathrm{P}$ and $\mathrm{S}$.

Since, higher grain and stover yield was recorded at $125 \%$ recommended $\mathrm{K}_{2} \mathrm{O}$ as potassium schoenite and was in accordance with higher uptake of N, P and K. Similarly, Fu and Chen (1982) indicated that uptake of $\mathrm{K}$ increased with increasing yield of maize and the soil fertility.

\section{Relationship between levels of $\mathrm{K}$ and $\mathrm{N}, \mathbf{P}$ and $K$ uptake by maize crop}

Total N, P and K uptake by maize crop showed positive and linear association with levels of $\mathrm{K}$ application and is described in equation 1 to 3 .

$y=0.7109 x+78.591 ; R^{2}=0.9866----(1)$

Total $\mathrm{N}$ uptake was (Figure 1 and equation 1) influenced to an extent of $98.66 \%$ by the levels of $\mathrm{K}$ application, which indicates that increase in the levels of $\mathrm{K}$ application there will be an increase in total uptake of $\mathrm{N}$ and it has positive relation on levels of $\mathrm{K}$ application.

$\mathrm{y}=0.1746 \mathrm{x}+18.943 ; \mathrm{R}^{2}=0.9827----(2)$

A positive quadratic association between total uptake of $\mathrm{P}$ by maize crop and levels of $\mathrm{K}$ application was observed (Figure 2). The fitted regression model could be described by the equation no. 2. Total uptake of $\mathrm{P}$ is explained by $98.27 \%$ dependency on the levels of $\mathrm{K}$ applied.

$y=0.5856 x+60.479 ; R^{2}=0.9926----(3)$

Total uptake of $\mathrm{K}$ increased linearly with the increase in levels of $\mathrm{K}$ application (Figure 3). The fitted regression model was described by the equation 3. Where, variability in total uptake of $\mathrm{K}$ by maize crop was explained to an extent of $99.26 \%$ by the varied levels of $\mathrm{K}$ application. Increase in uptake of nutrient with increase in levels of $\mathrm{K}$ application on low $\mathrm{K}$ soils in the present studies implies that inadequate supply or absence of any one major nutrient to the crop would result in imbalance supply of other nutrient elements and consequent reduction in yield, nutrient use efficiency and uptake (Anonymous, 2006).

As such, with increase in levels of $\mathrm{K}$ application there was an increase in uptake of other nutrients viz., nitrogen and phosphorus was due to synergetic influence and translocation of other nutrients by the applied K (Sanjeev Kumar et al., 2015). Therefore, positive and linear relation was observed between total uptake of $\mathrm{N}, \mathrm{P}$ and $\mathrm{K}$ by maize crop with increased levels of $\mathrm{K}$ application.

This study clearly indicated that the $\mathrm{K}$ level can be increased $25 \%$ higher than the RDF (40 kg $\mathrm{ha}^{-1}$ ) for maize crop for getting higher yield.

Similarly, among the sources potassium schoenite was found to be the best source, where higher amount of total uptake of N, P and $\mathrm{K}$ was recorded compared to the other sources and there was linear quadratic relationship between nutrient uptake and levels of K applied.

So, application of $125 \% \quad \mathrm{~K}_{2} \mathrm{O}\left(50 \quad \mathrm{~kg} \mathrm{ha}^{-1}\right)$ through potassium schoenite is beneficial not only for better nutrient uptake but also for getting higher yield, in low $\mathrm{K}$ soils of Eastern dry zone of Karnataka.

\section{References}

Mengel, K. and Kirkby, E. A., 1978, Principles of plant nutrition. $4^{\text {th }}$ edtion, International potash institute Worblaufen-Berne, Switcherland. Pp. 31-33.

Sarkar, G. K., Debnath, A., Chattopadhyay, A. P. and Sanyal, S. K., 2014, Depletion of soil potassium under exhaustive cropping in Inceptisols and Alfisols. Comm. Soil Sci. Pl. Anal., 45: 61-72.

Kusro, P. S., Singh, D. P., Deepak Kumar and Manish, A., 2014. Response of potassium in inceptisol under cropping pattern system after fourth crop cycle. American 
International Journal of Research in Formal, Appl. Nat. Sci., 5(1): 81-82.

Piper, C. S., 1966, Soil and plant analysis. Hans Publishers. Bombay

Jackson, M. L., 1973, Soil Chemical Analysis. Prentice Hall of India Private Limited, New Delhi.

Gomez, K. A. and Gomez, A. A., 1984, Statistical Procedures for Agric. Res. $2^{\text {nd }}$ Ed. John Wiley \& Sons, New York.

Arun Kumar, M. A., Gali, S. K. and Hebsur, N. S., 2007, Effect of different levels of NPK on growth and yield parameters of sweet corn. Karnataka J. Agric. Sci., 20(1): 4143.

Manjunatha., 2011, Main and residual effect of graded levels of potassium with or without magnesium and sulphur on yield and soil nutrient status in rice-cowpea system, M.Sc.(Agri.) Thesis, Univ. Agri. Sci., Bangalore.

Channabasamma. A., Habsur, N. S., Bangaremma, S. W. and Akshaya, M. C., 2013, Effect of nitrogen and sulphur levels and ratios on growth and yield of maize. Molecular Pl. Breeding, 4(37): 292-296.

Ujwala Ranade. M., 2011, Interaction of micronutrients with major nutrients with special reference to potassium. Karnataka J. Agric. Sci., 24(1): 106-109.

Sanjeev Kumar., Shivadhar, Ashok Kumar and Dileep Kumar., 2015, Yield and nutrient uptake of maize (Zea mays)-wheat (Triticum aestivum) cropping system as influenced by integrated potassium management. Indian J. Agron., 60(4): 511-515.
Bagavatiammal, V. and Muthiah, N. D., 1995, Studies on the application of potassium and organic manures on the uptake of nutrients in red soil. Madras Agric. J., 82(1): 55-57.

Sudhir, K., Mariswamygowda, S. M. And Srikanth, K., 1998, Long-term effect of continuous cropping and fertilizer use on sustenance of productivity and fertility of an Alfisols under irrigated finger millethybrid maize system. In: Long-term fertility management through integrated plant nutrient system, AICRP on LTFE. ICAR, pp. 139-145.

Ahmad Alias Haji, m., Bukhsh, A., Riaz Ahmad, Javaid Iqbal, SafdarHussain., Atique Ur rehman and Ishaque, M., 2011, Potassium application reduces bareness in different maize hybrids under crowding stress conditions. Pak. J. Agri. Sci., 48 (1): 31-38

Shubhangi, J. D., Patil, V. D. and Mamta, J. P., 2014, Effect of various levels of phosphorus and sulphur on yield, plant nutrient content, uptake and availability of nutrients at harvest stages of soybean [Glycine max (L.)]. Int. J. Curr. Microbiol, App. Sci. 3 (12): 833-844.

Fu, Y. C. and Chen, G. P., 1982, Studies on fertilizer requirements and dry matter accumulation on summer maize. Agronomica Sinica, 8(1): 1-8.

Anonymous, 2006, Annual progress report. AICRP on Long Term Fertilizer Experiments to study the changes in soil quality, crop productivity and sustainability. IISS, Bhopal.

\section{How to cite this article:}

Sidharam Patil, P.K. Basavaraja, V.R. Ramakrishna Parama, T. Chikkaramappa and Sheshadri, T. 2017. Effect of Different Sources and Levels of K on Maize (Zea mays L.) Yield, Nutrient Content and Uptake by Maize Crop in Low $\mathrm{K}$ Soils of Eastern Dry Zone of Karnataka. Int.J.Curr.Microbiol.App.Sci. 6(8): 577-587. doi: https://doi.org/10.20546/ijcmas.2017.608.075 